\title{
Alleviation of salt stress by Halomonas sp. and osmolytes in Zea mays
}

\author{
Aisha Waheed Qurashi* and Anjum Nasim Sabri \\ Department of Microbiology and Molecular Genetics, University of the Punjab, Quaid-i-azam Campus, Lahore, Pakistan- \\ 54590 . \\ Accepted 21 September, 2011
}

\begin{abstract}
Level of osmolytes accumulation to a variable extent in bacteria and plants is associated with the salt tolerance. We hypothesized that two previously isolated salt tolerant strains, Halomonas meridiana (PAa6) and Halomonas aquamarina (RT2), found to stimulate the growth of wheat also have the ability to accumulate osmolytes. These strains can favour plant growth due to their endogenous osmolyte accumulation. To ascertain this, strains were checked for osmolyte accumulation under salt stress. Growth and osmolyte accumulation of inoculated and non-inoculated seeds of the economically significant plant, Zea mays Var. EV.90, was recorded. Seeds were sown in soil supplemented with and without exogenous proline and glycine betaine and salt stress for 15 days. The results show that strains can accumulate osmolytes (preferably glycine betaine) at higher salt stress. Inoculation and exogenous osmolytes $(10 \mathrm{mM})$ improved the plant growth at higher salt stress. However, concurrent application had an amplified effect. Endogenous level of osmolyte (preferably glycine betaine) on the fresh weight basis was significantly higher and improved bacterial and plant growth under stress. We conclude that accumulation of osmolytes in bacteria and plants significantly stimulated growth and protect them from the adverse effect of salt stress. This can be an alternate economic approach to increase crop productivity.
\end{abstract}

Key words: Osmolyte, glycine betaine, proline, salt stress.

\section{INTRODUCTION}

Dependence on multipurpose cereal crop maize is growing to a large extent throughout the world, while putting together all its uses. Sensitivity of maize to salt stress is of major concern for farmers all over the world (Maas et al., 1983; Jadhav et al., 2010). It is noteworthy for Pakistan (Nasim et al., 2008) and other countries where increasing population has markedly established the food security problems. Supporting a superior portion of the world's economy, it is vital to amplify maize yields even under the hostile conditions of salt stress. Salt stress, either due to badly chosen irrigation exercise, no drainage, poor soil management and higher evapotranspiration causes build-up of excess salts in the soil which is adverse to the crops yield (Afrasayab et al., 2010). Accumulation of osmolytes in bacteria and plants

\footnotetext{
${ }^{*}$ Corresponding author. E-mail: aieshawaheed@yahoo.com.
}

is a stress-related phenomenon (Canovas et al., 1998).Although, much salt tolerant plants accumulate osmolytes in response to salt stress due to their genetic and biochemical advantage, they are still plants that can do this according to their metabolic capacity. Thus, higher accumulation of these osmolytes in response to salt stress is of prime importance in stress adaptation. The safer use of indigenous microorganisms for plant yield is among one of the cost effective technologies (Afrasayab and Hasnain, 2000a; Ahmed and Hasnain, 2010).

In this study, we worked on a number of bacteria isolated from the close vicinity of plants of saline soils in Pakistan; PAa6 from phylloplane of Achyranthus aspera (Afrasayab and Hasnain, 2000b) and Halomonas aquamarina (RT2) from rhizoplane of Mazus specie (Mirza et al., 1998). These have the innate ability to thrive under salt stress to a variable extent where existence of non-halophilic bacteria is difficult. These strains have also been reported to improve Triticum aestivum plant growth 
by increasing their auxin, alkaline phosphatases, acid phosphatase and proteins content (Mirza et al., 1998; Afrasayab and Hasnain., 2000b; Mehr et al., 2002; Afrasayab et al., 2010; Ahmed and Hasnain, 2010). Increased endogenous accumulation of osmolytes (proline, glycine betaine, ectoine, hydroxyectoine, choline and other amino acids) provide a salt tolerance to organisms (Roberts, 2005). Role of compatible solutes to bacteria as well as to plants resulted in improving their stress resistance (Canovas et al., 1998; Roberts, 2005; Vyrides and Stuckey, 2009). Other reports also highlight the beneficial impact of microorganisms isolated from stressed soil on plant growth (Sadowsky, 2005; Hirsch, 2010; Karlidag et al., 2011). From previous studies, considerable successful attempts have been made to ameliorate soil salinity with exogenous osmolytes like proline and glycine betaine.

With the aim of taking any measure toward economic and sustainable agriculture, we used salt tolerant bacteria and checked the potential for plant growth promotion for present research work. In our work, we studied growth and endogenous osmolyte accumulation from plants and bacterial point of view. Moreover, the effect of bacterial inoculation on salt sensitive maize plants were also compared with plants treated with exogenously applied osmolytes and varying salt concentrations.

\section{MATERIALS AND METHODS}

\section{Growth conditions and osmolytes accumulation of bacterial strains}

Previously isolated and characterized bacterial strains $H$. meridiana (PAa6) (accession no. HQ256515) (Afrasayab and Hasnain, 2000b) and $H$. aquamarina (RT2) (accession no. HQ107978) (Mirza et al., 1998), were used for the current study. Strains were maintained routinely in LB agar (Gerhardt et al., 1994) supplemented with 0.5 $\mathrm{M} \mathrm{NaCl}$.

To study the effect of different $\mathrm{NaCl}$ concentrations $(0.5,1,1.5,2$ and $2.5 \mathrm{M}$ ) on the growth and osmolyte accumulation of bacterial strains, $10 \mathrm{ml}$ LB (Gerhardt et al., 1994) and M9 broth (Kahn et al., 1979) added with respective molar concentrations of $\mathrm{NaCl}$ were poured in test tubes and autoclaved. 24-h old bacterial suspension (absorbance adjusted $0.3 \mathrm{~A}$ at $600 \mathrm{~nm} ; 10^{8} \mathrm{ml}^{-1}$ CFUs) was inoculated in each test tube containing $L$ broth and M9 media. One test tube in each case was used as a control. All the test tubes were incubated at $37^{\circ} \mathrm{C}$ for $24 \mathrm{~h}$ at $150 \mathrm{rpm}$ on the shaker (orbital incubator Model I-4000 serial number 104 A IRMECO GmbH, Goesthacht/Germany) at $\mathrm{pH}$ 8.0. After $24 \mathrm{~h}$ incubation, bacterial growth was recorded by measuring absorbance $\left(\mathrm{OD}_{600}\right)$ using the spectro-photometer (Model S-300 DL, R \& M marketing, and England).

Also, bacterial cells were harvested from cultures after $24 \mathrm{~h}$ by centrifugation (2000 rpm for $5 \mathrm{~min}$ ), weighed and suspended in $1 \mathrm{ml}$ sterile distilled water. Cells were used for determination of endogenous proline following the method of Tonon et al. (2004). The level of endogenous glycine betaine and choline was determined following the method of Grieve and Grattan (1983). Calibration of proline and glycine betaine was made using reference L-proline and glycine betaine, respectively. The experiments were carried out with three replicates and mean and standard errors of means were calculated. In all the Figures, the values are represented with standard errors of the means.
Inoculation of plants, harvesting and biochemical analysis

For inoculum preparation, bacterial strains were incubated overnight $\left(0.5 \mathrm{M} \mathrm{NaCl}\right.$ stress, $\mathrm{pH}$ and temperature $\left.37^{\circ} \mathrm{C}\right)$ at $150 \mathrm{rpm}$ shaker in LB medium. Overnight cultures were centrifuged and bacterial pellet was suspended in sterile water. Optical density of this suspension was measured on spectrophotometer at $600 \mathrm{~nm}$. To ensure the equal cell population of each bacterial strain, optical density of the suspension was adjusted to $10^{8}$ cells $\mathrm{ml}^{-1}$. Healthy and certified seeds of Zea mays Var. EV-90 were obtained from Punjab Seed Corporation, Lahore. Pakistan and used in experiments. Seeds were disinfected with $0.1 \% \mathrm{HgCl}_{2}$ solution for 10 minutes and followed by five to six times rinsing with sterile water to remove all traces of $\mathrm{HgCl}_{2}$ completely. After surface sterilezation, seeds were inoculated with $10 \mathrm{ml}$ of bacterial suspension for 30 minutes. Uninoculated seeds were sown as control treatments. For control, seeds were soaked in sterile water of same volume and for the same period of time in disposable sterile plastic pots filled with $120 \mathrm{~g}$ sieved and sterilized garden soil with 95\% water- holding capacity.

After seed were sown, equal volume of salt solution was added to each pot so that the final concentrations of the $\mathrm{NaCl}$ in the pots were 50,100 and $200 \mathrm{mM}$ per gram weight of soil. Pots without any salt solution $(0 \mathrm{mM} \mathrm{NaCl})$ were treated as control. Similarly, filter sterilized solutions of proline and glycine betaine were added to each pot making the final concentrations $10 \mathrm{mM}$ per gram weight of soil. To prevent fluctuations in salt concen-trations of soil, pots were watered with care so that salt or soil was not drained out of the pot. Seeds were allowed to germinate in the dark in a growth chamber at $28 \pm 30^{\circ} \mathrm{C}$ for three days, and germination was recorded daily. After seed germination, pots were transferred to light intensity of 10 Klux, photoperiod of 16 - $h$ light/dark and temperature $37^{\circ} \mathrm{C} \pm 1$. The seedlings were harvested after 15 days and different growth parameters including germination, shoot length, root length, fresh weight, dry weight and Chlorophyll ( $\mathrm{a}, \mathrm{b}$ and carotenoid content) were recorded. Endogenous proline from harvested plants was determined following Tonon et al. (2004); endogenous glycine betaine and choline were determined following Grieve and Grattan (1983), while chlorophyll a, b and carotenoid contents of the plants were determined following Lichtenthaler and Wellburn (1983). The experiment was carried out in three replicates. Mean and standard errors of means were calculated, and the difference between the means was tested using the least significant difference test $(p<0.05)$.

\section{RESULTS}

\section{Determination of osmolyte accumulation in bacterial strains}

The effect of varying concentrations of $\mathrm{NaCl}$ on the growth of the bacterial strains was recorded. With increasing salt concentrations, decrease in bacterial growth was observed. Growth was comparatively better in LB as compared to M9 medium (Figure 1). Optimum growth of strain $H$. meridiana (PAa6) and $H$. aquamarina (RT2) was observed at $0.5 \mathrm{M} \mathrm{NaCl}$ stress after which growth decreased for both strains in LB and M9 media, respectively. Although, both strains of $H$. meridiana (PAa6) and $H$. aquamarina (RT2) showed a comparable growth profile in LB and M9 media, however, growth was comparatively less in M9 media compared to LB (Figure 1). Unlike growth pattern, cellular osmolyte accumulation 


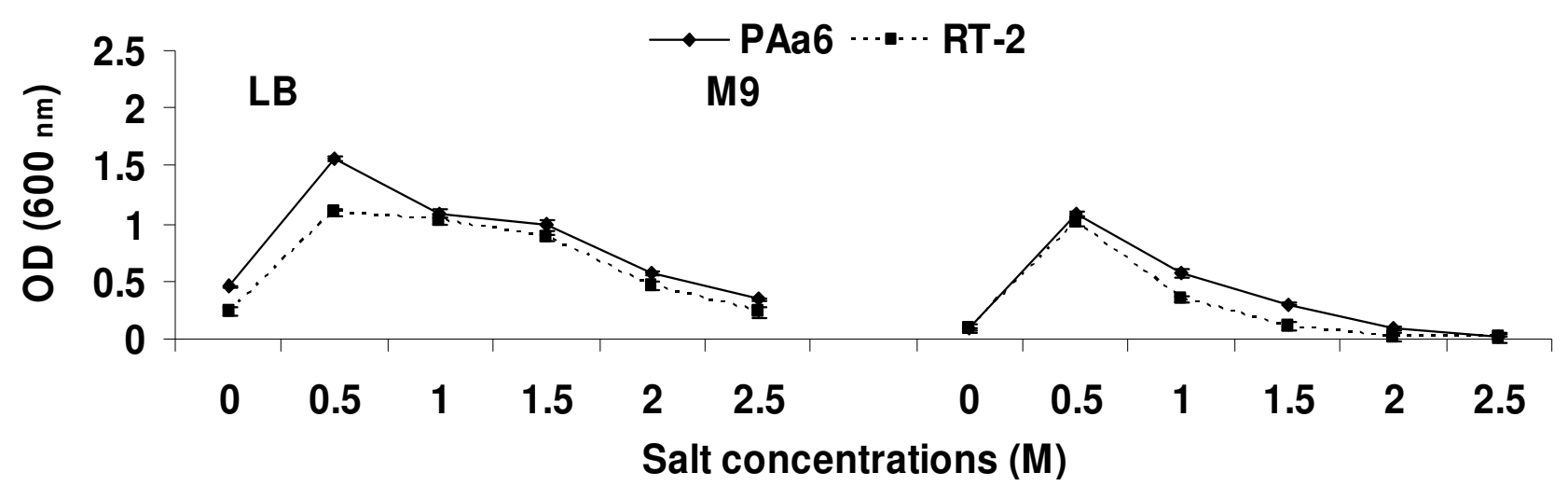

Figure 1. Effect of varying $\mathrm{NaCl}$ concentrations $(\mathrm{M})$ on bacterial growth in $\mathrm{LB}$ and $\mathrm{M9}$ media.

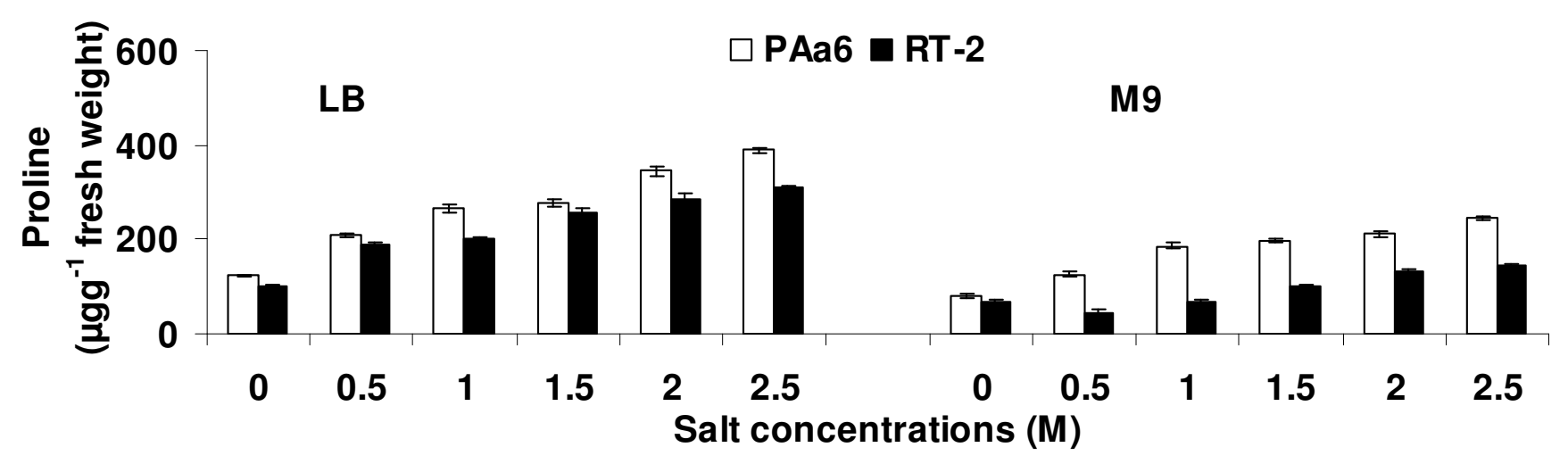

Figure 2. Effect of varying $\mathrm{NaCl}$ concentrations $(\mathrm{M})$ on endogenous proline ( $\mu \mathrm{gg}^{-1}$ fresh weight of bacterial strains) accumulation in LB and M9 media.

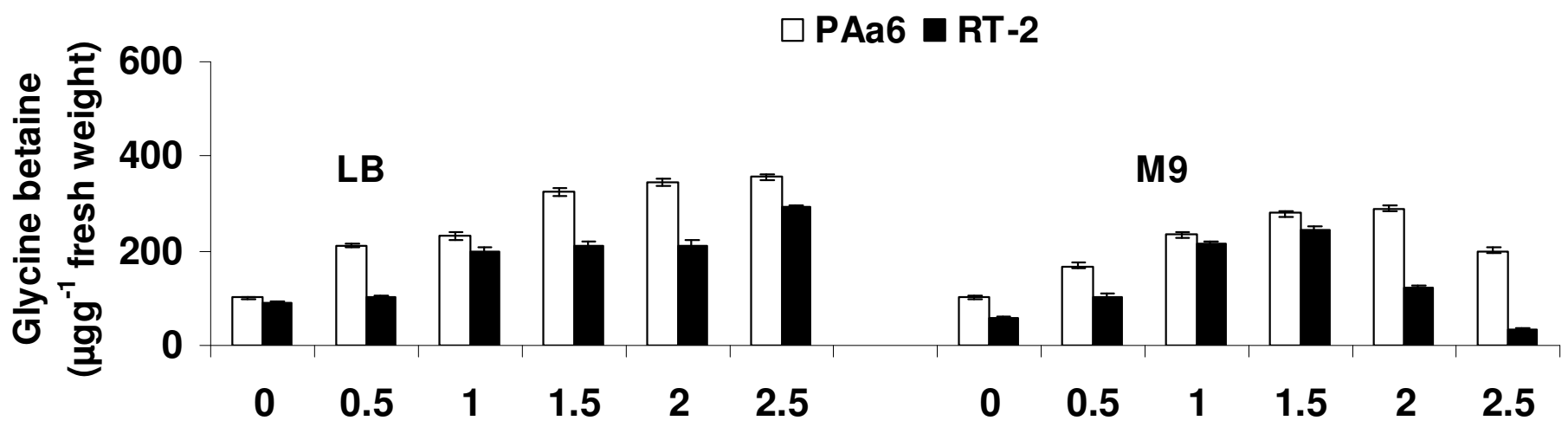

Figure 3. Effect of varying $\mathrm{NaCl}$ concentrations $(\mathrm{M})$ on endogenous glycine betaine $\left(\mu \mathrm{gg}^{-1}\right.$ fresh weight of bacterial strains) accumulation in LB and M9 media.

on the fresh weight basis tends to increase at increasing salt stress. However, in general, accumulation of choline (Figure 4) was less as compared to proline (Figure 2) and glycine betaine (Figure 3 ) osmolytes. At $0 \mathrm{mM}$ salt stress, accumulation of osmolytes was lower (Figures 2 to 4 ). $H$. meridiana (PAa6) was found to be more efficient in accumulating both osmolytes than $H$. aquamarina (RT2) strain. Accumulations of glycine betaine (Figure 3) were higher in $\mathrm{H}$. aquamarina (RT2) at 0 and $0.5 \mathrm{M} \mathrm{NaCl}$ supplemented media. Maximum choline accumulation was observed at $1.5 \mathrm{M}$ to $2.5 \mathrm{M} \mathrm{NaCl}$ stress in LB medium. 


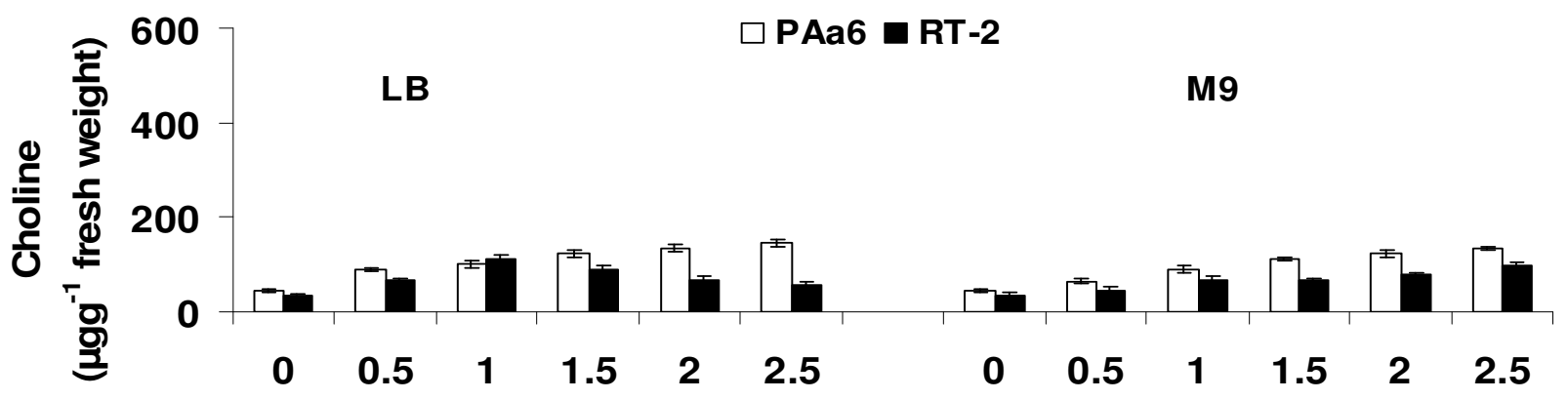

Figure 4. Effect of varying $\mathrm{NaCl}$ concentrations $(\mathrm{M})$ on endogenous choline ( $\mu \mathrm{gg}^{-1}$ fresh weight of bacterial strains) accumulation in LB and M9 media.

Table 1. Effect of varying $\mathrm{NaCl}$ concentrations (mM) and exogenously applied osmolytes (proline $10 \mathrm{mM}$ and glycine betaine $10 \mathrm{mM}$ ) on germination (\%) of Zea mays $\mathrm{Var}$. EV.90.

\begin{tabular}{|c|c|c|c|c|c|c|c|c|c|c|c|c|}
\hline \multirow{3}{*}{ Treatment } & \multicolumn{12}{|c|}{ Germination (\%) } \\
\hline & \multicolumn{4}{|c|}{ Salt concentration (mM) } & \multicolumn{4}{|c|}{ Salt concentration $(\mathrm{mM})+10 \mathrm{mM}$ proline } & \multicolumn{4}{|c|}{ Salt concentration $(\mathrm{mM})+10 \mathrm{mM}$ glycine betaine } \\
\hline & $\mathbf{0}$ & 50 & 100 & 200 & 0 & 50 & 100 & 200 & 0 & 50 & 100 & 200 \\
\hline Control & $100 \pm 1.23$ & $34 \pm 13$ & $28 \pm 12$ & $17 \pm 9$ & $75 \pm 0.89$ & $85 \pm 1.33$ & $65 \pm 1.32$ & $65 \pm 0.99$ & $65 \pm 0.98$ & $55 \pm 1.11$ & $55 \pm 2.22$ & $45 \pm 1.29$ \\
\hline PAa6 & $100 \pm 1.23$ & $78 \pm 15$ & $56.09 \pm 23$ & $53 \pm 14$ & $75 \pm 1.99$ & $85 \pm 1.45$ & $90 \pm 2.33$ & $75 \pm 1.24$ & $85 \pm 0.98$ & $75 \pm 2.22$ & $75 \pm 2.53$ & $60 \pm 1.34$ \\
\hline RT-2 & $100 \pm 2.23$ & $56 \pm 20$ & $48.56 \pm 23$ & $47 \pm 12$ & $65 \pm 1.23$ & $65 \pm 2.30$ & $40 \pm 2.43$ & $55 \pm 1.45$ & $75 \pm 1.23$ & $65 \pm 1.23$ & $45 \pm 2.33$ & $59 \pm 1.92$ \\
\hline
\end{tabular}

LSD at $p=0.05$

Between strains 35.5812 .1316 .32

Between treatments 30.818 .5711 .54

\section{Effect of bacterial inoculation on growth parameters of Zea. mays seedlings}

Severe reduction in germination of seedlings (Table 1 ) at $\mathrm{NaCl}$ stress was observed especially at $200 \mathrm{mM} \mathrm{NaCl}(83 \%)$ as compared to non inoculated control plants grown without $\mathrm{NaCl}$ stress. However, noteworthy increase (211\%) was observed with $H$. meridiana (PAa6) and $H$. aquamarina (RT2) (176\%) at $200 \mathrm{mM} \mathrm{NaCl}$ stress. Exogenous application of $10 \mathrm{mM}$ proline and $10 \mathrm{mM}$ glycine betaine also improved seed germination. However, bacterial inoculation along with exogenous osmolytes significantly increased the germination. However, addition of proline with bacterial inoculation $H$. aquamarina (RT2) reduced the germination with and without salt stress compared to non-inoculated controls.

Salt stress resulted in the maximum reduction in shoot length (33\%) and root length (38\%) at 200 $\mathrm{mM} \mathrm{NaCl}$ stress, respectively over noninoculated plants at $0 \mathrm{mM} \mathrm{NaCl}$ stress. Bacterial inoculation caused an increment (58.3 with PAa6 and 17\% with RT2) in shoot length (Table 2) at no salt stress. Both strains $H$. meridiana (PAa6) and $H$. aquamarina (RT2) showed maximum $40 \%$ increment at $50 \mathrm{mM} \mathrm{NaCl}$ stress. Similar response was seen in case of root length (Table 3); strain
H. meridiana (PAa6) showed $46 \%$ increment, while $H$. aquamarina (RT2) showed $39 \%$ increment at $0 \mathrm{mM} \mathrm{NaCl}$ stress. Exogenous application of glycine betaine improved root length compared to exogenous proline. Root length also increased (87\%) with exogenous proline and (137\%) with glycine betaine at $200 \mathrm{mM}$ salt stress compared to non-osmolyte supplemented control plants. With the combination of exogenous osmolytes and bacterial strains, the effect of glycine betaine was slightly higher than proline. Addition of proline resulted in $30 \%$ and $19 \%$ increase in shoot length with $H$. meridiana (PAa6) and $H$. aquamarina (RT2) strain, 
Table 2. Effect of varying $\mathrm{NaCl}$ concentrations (mM) and exogenously applied osmolytes (proline $10 \mathrm{mM}$ and glycine betaine $10 \mathrm{mM}$ ) on Shoot length (cm) of $Z$. mays $\mathrm{Var}$. EV.90.

\begin{tabular}{|c|c|c|c|c|c|c|c|c|c|c|c|c|}
\hline \multirow{3}{*}{ Treatment } & \multicolumn{12}{|c|}{ Shoot length $(\mathrm{cm})$} \\
\hline & \multicolumn{4}{|c|}{ Salt concentration (mM) } & \multicolumn{4}{|c|}{ Salt concentration $(\mathrm{mM})+10 \mathrm{mM}$ proline } & \multicolumn{4}{|c|}{ Salt concentration $(\mathrm{mM})+10 \mathrm{mM}$ glycine betaine } \\
\hline & 0 & 50 & 100 & 200 & 0 & 50 & 100 & 200 & 0 & 50 & 100 & 200 \\
\hline Control & $12 \pm 0.45$ & $10 \pm 0.78$ & $8 \pm 0.89$ & $8 \pm 0.89$ & $10 \pm 0.89$ & $14.4 \pm 0.45$ & $13 \pm 0.79$ & $10 \pm 0.56$ & $18 \pm 0.67$ & $16 \pm 0.68$ & $15 \pm 1.89$ & $15 \pm 3.89$ \\
\hline PAa6 & $19 \pm 0.89$ & $14 \pm 0.98$ & $12 \pm 0.89$ & $10 \pm 0.89$ & $20 \pm 0.63$ & $18 \pm 0.56$ & $14 \pm 0.34$ & $13 \pm 0.89$ & $25 \pm 0.56$ & $23.5 \pm 0.89$ & $20 \pm 2.0$ & $18.5 \pm 2.09$ \\
\hline RT-2 & $14 \pm 0.88$ & $14 \pm 0.34$ & $11 \pm 1.09$ & $10.9 \pm 0.98$ & $22 \pm 0.34$ & $17 \pm 0.23$ & $15 \pm 0.34$ & $11.9 \pm 0.45$ & $21 \pm 0.49$ & $19.12 \pm 0.78$ & $16.5 \pm 1.79$ & $13.5 \pm 2.23$ \\
\hline
\end{tabular}

LSD at $p=0.05$

Between strains 2.0463 .861 .738

Between treatments 1.7723 .351 .505

Table 3. Effect of varying $\mathrm{NaCl}$ concentrations (mM) and exogenously applied osmolytes (proline $10 \mathrm{mM}$ and glycine betaine $10 \mathrm{mM}$ ) on root length (cm) of $Z$. mays $\mathrm{Var}$. EV90.

\begin{tabular}{|c|c|c|c|c|c|c|c|c|c|c|c|c|}
\hline \multirow{3}{*}{ Treatment } & \multicolumn{12}{|c|}{ Root length (cm) } \\
\hline & \multicolumn{4}{|c|}{ Salt concentration (mM) } & \multicolumn{4}{|c|}{ Salt concentration $(\mathrm{mM})+10 \mathrm{mM}$ proline } & \multicolumn{4}{|c|}{ Salt concentration $(\mathrm{mM})+10 \mathrm{mM}$ glycine betaine } \\
\hline & 0 & 50 & 100 & 200 & 0 & 50 & 100 & 200 & 0 & 50 & 100 & 200 \\
\hline Control & $13 \pm 0.89$ & $12 \pm 0.34$ & $8 \pm 0.78$ & $8 \pm 1.09$ & $22 \pm 0.34$ & $19 \pm 0.78$ & $19 \pm 0.65$ & $15 \pm 0.99$ & $26 \pm 0.76$ & $23 \pm 0.67$ & $21 \pm 0.29$ & $19 \pm 0.57$ \\
\hline PAa6 & $19 \pm 0.82$ & $14 \pm 0.24$ & $13 \pm 0.79$ & $11 \pm 0.59$ & $25 \pm 0.32$ & $21 \pm 0.34$ & $18 \pm 0.56$ & $16 \pm 0.34$ & $29 \pm 0.68$ & $25 \pm 0.54$ & $28 \pm 0.38$ & $25 \pm 0.78$ \\
\hline RT-2d & $18 \pm 0.56$ & $13 \pm 0.89$ & $10 \pm 1.67$ & $9 \pm 0.78$ & $21 \pm 0.89$ & $21 \pm 0.89$ & $20 \pm 0.89$ & $18 \pm 0.79$ & $26 \pm 0.98$ & $23.5 \pm 0.59$ & $29 \pm 0.56$ & $20 \pm 2.39$ \\
\hline
\end{tabular}

LSD at $p=0.05$

Between strains 2.0596 .352 .22

Between treatments 1.7865 .5061 .928

respectively at $200 \mathrm{mM} \mathrm{NaCl}$ stress, compared to non-inoculated control plants grown at same treatment. Combination of strain $H$. meridiana (PAa6) and exogenously applied glycine betaine also increased the root length by $33 \%$ at $100 \mathrm{mM}$ and $31.5 \%$ at $200 \mathrm{mM} \mathrm{NaCl}$ compared to noninoculated control plants.

In addition, maximum reduction (96\%) in fresh weight (Table 4) was observed at $200 \mathrm{mM} \mathrm{NaCl}$ stress compared to control plant. However, both strains improved fresh and dry weight (Table 5) over noninoculated control plants at same stress. Strain $H$. meridiana (PAa6) showed $144 \%$ increase and strain $H$. aquamarina (RT2) showed
$77.7 \%$ increase in fresh weight, over respective non-inoculated control plants at $0 \mathrm{mM}$ salt stress. Both strains $H$. meridiana (PAa6) and $H$. aquamarina (RT2) showed upper limit increment in fresh weight (1100 and $1111 \%)$ and dry weight $(261 \%$ and $206 \%)$ at $200 \mathrm{mM} \mathrm{NaCl}$ stress, respectively. Bacterial inoculation and exogenously applied osmolytes showed maximum increase in fresh weight and dry weight of plant at $100 \mathrm{mM} \mathrm{NaCl}$ stress. Exogenously applied glycine betaine showed significant increase in the dry weight values at $200 \mathrm{mM} \mathrm{NaCl}$ stress.

Furthermore, salt stress improved the plant growth by improving chlorophyll contents of plants
(Table 6). Salt stress resulted in $40 \%$ increase at $50 \mathrm{mM} \mathrm{NaCl}$, and 33 and $33 \%$ reduction in Chlorophyll a contents at 100 and $200 \mathrm{mM} \mathrm{NaCl}$ stress, respectively over noninoculated plants at 0 $\mathrm{mM} \mathrm{NaCl}$ stress. Similarly, $18 \%, 49 \%$ and $69 \%$ reduction at 50,100 and $200 \mathrm{mM} \mathrm{NaCl}$ stress in chlorophyll b contents (Table 7) was recorded over noninoculated plants at $0 \mathrm{mM} \mathrm{NaCl}$ stress. Carotenoid contents (Table 8) increased 0.47 at $50 \mathrm{mM}$ and $43.5 \%$ at $100 \mathrm{mM}$, respectively over noninoculated plants at $0 \mathrm{mM} \mathrm{NaCl}$ stress. No change was however observed for chlorophyll b contents of inoculated plants at $200 \mathrm{mM} \mathrm{NaCl}$ stress. Inoculation of Halomonas meridiana 
Table 4. Effect of varying $\mathrm{NaCl}$ concentrations (mM) and exogenously applied osmolytes (proline $10 \mathrm{mM}$ and glycine betaine $10 \mathrm{mM}$ ) on fresh weight (g) of $Z$. mays $\mathrm{Var}$. EV90.

\begin{tabular}{|c|c|c|c|c|c|c|c|c|c|c|c|c|}
\hline \multirow{3}{*}{ Treatment } & \multicolumn{12}{|c|}{$\begin{array}{r}\text { Fresh weight }(\mathrm{g}) \\
\end{array}$} \\
\hline & \multicolumn{4}{|c|}{ Salt concentration (mM) } & \multicolumn{4}{|c|}{ Salt concentration $(\mathrm{mM})+10 \mathrm{mM}$ proline } & \multicolumn{4}{|c|}{ Salt concentration $(\mathrm{mM})+10 \mathrm{mM}$ glycine betaine } \\
\hline & 0 & 50 & 100 & 200 & 0 & 50 & 100 & 200 & 0 & 50 & 100 & 200 \\
\hline Control & $2.7 \pm 0.12$ & $1.8 \pm 0.09$ & $1.8 \pm 0.12$ & $0.09 \pm 0.01$ & $3.8 \pm 0.56$ & $2.8 \pm 0.19$ & $1.9 \pm 0.89$ & $1.5 \pm 0.89$ & $4.6 \pm 0.67$ & $3.6 \pm 0.89$ & $2.5 \pm 0.89$ & $2.0 \pm 1.89$ \\
\hline PAa6 & $6.6 \pm 0.15$ & $5.0 \pm 0.12$ & $3.8 \pm 0.12$ & $1.08 \pm 0.02$ & $6.9 \pm 0.65$ & $4.5 \pm 0.52$ & $4.5 \pm 0.56$ & $2.9 \pm 0.56$ & $10.9 \pm 2.09$ & $7.1 \pm 0.22$ & $6.3 \pm 1.89$ & $4.5 \pm 0.89$ \\
\hline RT-2 & $4.8 \pm 0.89$ & $2.8 \pm 0.23$ & $2.0 \pm 0.09$ & $1.09 \pm 0.09$ & $4.5 \pm 0.19$ & $3.5 \pm 0.83$ & $2.0 \pm 0.56$ & $2.5 \pm 0.34$ & $10.9 \pm 1.98$ & $5.4 \pm 0.67$ & $5.4 \pm 3.78$ & $3.9 \pm 0.67$ \\
\hline
\end{tabular}

LSD at $\mathrm{p}=0.05$

Between strains 3.100 .9951 .34

Between treatments 2.680 .7030 .94

Table 5. Effect of varying $\mathrm{NaCl}$ concentrations (mM) and exogenously applied osmolytes (proline $10 \mathrm{mM}$ and glycine betaine $10 \mathrm{mM}$ ) on dry weight ( $\mathrm{g}$ ) of $Z$. mays $\mathrm{Var}$. EV90.

\begin{tabular}{|c|c|c|c|c|c|c|c|c|c|c|c|c|}
\hline \multirow{3}{*}{ Treatment } & \multicolumn{12}{|c|}{ Dry weight (g) } \\
\hline & \multicolumn{4}{|c|}{ Salt concentration $(\mathrm{mM})$} & \multicolumn{4}{|c|}{$\begin{array}{l}\text { Salt concentration (mM) } \\
+10 \mathrm{mM} \text { proline }\end{array}$} & \multicolumn{4}{|c|}{$\begin{array}{l}\text { Salt concentration ( } \mathrm{mM} \text { ) } \\
+10 \mathrm{mM} \text { glycine betaine }\end{array}$} \\
\hline & 0 & 50 & 100 & 200 & 0 & 50 & 100 & 200 & 0 & 50 & 100 & 200 \\
\hline Control & $0.14 \pm 0.09$ & $0.2 \pm 0.01$ & $0.27 \pm 0.01$ & $0.34 \pm 0.03$ & $0.32 \pm 0.1$ & $0.29 \pm 0.1$ & $0.18 \pm 0.1$ & $0.13 \pm 0.02$ & $0.29 \pm 0.07$ & $0.19 \pm 0.09$ & $0.12 \pm 0.09$ & $0.09 \pm 0.09$ \\
\hline PAa6 & $0.8 \pm 0.09$ & $0.9 \pm 0.04$ & $1.06 \pm 0.1$ & $1.23 \pm 0.09$ & $1.2 \pm 0.15$ & $0.65 \pm 0.1$ & $0.49 \pm 0.5$ & $0.2 \pm 0.06$ & $0.98 \pm 2.0$ & $0.29 \pm 0.02$ & $0.25 \pm 0.09$ & $0.10 \pm 0.01$ \\
\hline RT-2 & $0.7 \pm 0.01$ & $0.8 \pm 0.09$ & $1.09 \pm 0.2$ & $1.04 \pm 0.23$ & $0.9 \pm 0.19$ & $0.45 \pm 0.2$ & $0.29 \pm 0.5$ & $0.19 \pm 0.04$ & $0.65 \pm 1.9$ & $0.23 \pm 0.07$ & $0.19 \pm 0.08$ & $0.19 \pm 0.01$ \\
\hline
\end{tabular}

LSD at $p=0.05$

Between strains 3.100 .5180 .274

Between treatments 2.680 .44880 .237

(PAa6) caused 97.5\%, $48 \%, 122 \%$ and $75 \%$ increase in chlorophyll a, $143 \%, 200 \%, 196 \%$ and $300 \%$ increase in chlorophyll b, while $143 \%$, $90.4 \%, 33 \%$ and $50 \%$ increase in carotenoid contents at $0,50,100$ and $200 \mathrm{mM} \mathrm{NaCl}$ stress, respectively.

Inoculation of $\mathrm{H}$. aquamarina (RT2) caused $97.5 \%, 48.2 \%, 122.5 \%$ and $50 \%$ increase in chlorophyll a contents while 22.4, 62.5, 100 and $229 \%$ increase in chlorophyll b contents at 0, 50, 100 and $200 \mathrm{mM} \mathrm{NaCl}$ stress, respectively over noninoculated plants. While 33.9 and $47.6 \%$ increase in carotenoid contents at 0 and $50 \mathrm{mM}$ $\mathrm{NaCl}$ stress, and $6 \%$ and $40 \%$ reduction in carotenoid content was observed in noninoculated plants at 100 and $200 \mathrm{mM} \mathrm{NaCl}$ stress, respectively. Application of exogenous glycine betaine $(10 \mathrm{mM})$ also increased $125 \%$ chlorophyll a at $100 \mathrm{mM} \mathrm{NaCl}$ stress while $433 \%$ increase in chlorophyll b was recorded as compared to control at $200 \mathrm{mM} \mathrm{NaCl}$ stress. Combination of seed inoculation with exogenously applied osmolytes increased both chlorophyll $a$ and $b$ contents.

Level of endogenous osmolytes accumulation was increased by (178\% and 123\%) with a combination of bacterial strain Halomonas meridiana (PAa6) and Halomonas aquamarina
(RT2), respectively and exogenous osmolytes at $200 \mathrm{mM} \mathrm{NaCl}$ stress.

Endogenous accumulation of proline (Table 9), glycine betaine (Table 10) and choline (Table 11) in seedlings were also found to be affected by bacterial inoculation. Increment in proline and glycine betaine accumulation was observed at increasing salt stress in non-inoculated control plants. However, this accumulation was further stimulated in inoculated plants. In non-inoculated plants $(134 \%)$ maximum increase in proline and boost $(286 \%)$ in glycine betaine at $200 \mathrm{mM} \mathrm{NaCl}$ was observed. Inocu-lation of strain $H$. meridiana (PAa6) caused maximum (156\%) increase at 200 


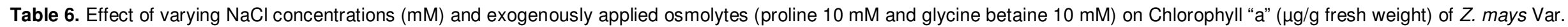
EV90.

\begin{tabular}{|c|c|c|c|c|c|c|c|c|c|c|c|c|}
\hline \multirow{3}{*}{ Treatment } & \multicolumn{12}{|c|}{ Chlorophyll "a" ( $\mu \mathrm{g} / \mathrm{g}$ fresh weight) } \\
\hline & \multicolumn{4}{|c|}{ Salt concentration $(\mathrm{mM})$} & \multicolumn{4}{|c|}{ Salt concentration $(\mathrm{mM})+10 \mathrm{mM}$ proline } & \multicolumn{4}{|c|}{ Salt concentration $(\mathrm{mM})+10 \mathrm{mM}$ glycine betaine } \\
\hline & 0 & 50 & 100 & 200 & 0 & 50 & 100 & 200 & 0 & 50 & 100 & 200 \\
\hline Control & $12 \pm 0.89$ & $16.78 \pm 0.89$ & $8 \pm 0.12$ & $8 \pm 0.89$ & $18 \pm 2.59$ & $18 \pm 1.78$ & $16 \pm 1.12$ & $14 \pm 0.69$ & $22 \pm 0.69$ & $22 \pm 2.89$ & $18 \pm 0.82$ & $16 \pm 0.79$ \\
\hline PAa6 & $23.7 \pm 0.34$ & $24.88 \pm 0.98$ & $17.8 \pm 0.9$ & $14 \pm 1.09$ & $32 \pm 1.94$ & $29 \pm 1.23$ & $20 \pm 1.9$ & $20 \pm 1.89$ & $39 \pm 1.84$ & $30 \pm 1.98$ & $25 \pm 0.99$ & $23 \pm 1.39$ \\
\hline RT-2 & $22.4 \pm 0.12$ & $23 \pm 0.99$ & $12 \pm 0.89$ & $12 \pm 1.34$ & $26 \pm 1.92$ & $22 \pm 1.78$ & $19 \pm 2.89$ & $19 \pm 0.94$ & $25 \pm 1.02$ & $25 \pm 1.99$ & $20 \pm 0.99$ & $18 \pm 1.24$ \\
\hline
\end{tabular}

LSD at $p=0.05$

Between strains 3.545 .2264 .872

Between treatments 3.063 .693 .445

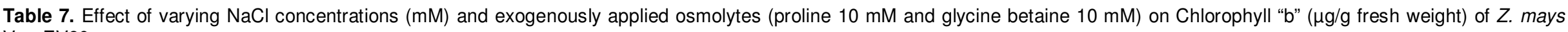
Var. EV90.

\begin{tabular}{|c|c|c|c|c|c|c|c|c|c|c|c|c|}
\hline \multirow{3}{*}{ Treatment } & \multicolumn{12}{|c|}{ Chlorophyll "b" ( $\mu \mathrm{g} / \mathrm{g}$ fresh weight) } \\
\hline & \multicolumn{4}{|c|}{ Salt concentration $(\mathrm{mM})$} & \multicolumn{4}{|c|}{ Salt concentration $(\mathrm{mM})+10 \mathrm{mM}$ proline } & \multicolumn{4}{|c|}{ Salt concentration $(\mathrm{mM})+10 \mathrm{mM}$ glycine betaine } \\
\hline & 0 & 50 & 100 & 200 & 0 & 50 & 100 & 200 & 0 & 50 & 100 & 200 \\
\hline Control & $9.8 \pm 0.23$ & $8 \pm 0.89$ & $5 \pm 0.89$ & $3 \pm 0.89$ & $10 \pm 0.45$ & $14 \pm 1.89$ & $12 \pm 2.89$ & $12 \pm 2.89$ & $23 \pm 0.78$ & $25 \pm 1.89$ & $18 \pm 0.69$ & $16 \pm 1.89$ \\
\hline PAa6 & $23.8 \pm 0.8$ & $24 \pm 1.00$ & $14.8 \pm 0.8$ & $12 \pm 0.12$ & $32 \pm 0.67$ & $29 \pm 2.00$ & $29 \pm 1.8$ & $20 \pm 1.12$ & $35.8 \pm 0.98$ & $32 \pm 2.00$ & $32 \pm 0.78$ & $30 \pm 2.12$ \\
\hline RT-2 & $12 \pm 0.12$ & $13 \pm 1.09$ & $10 \pm 1.09$ & $9.89 \pm 0.89$ & $29 \pm 1.09$ & $25 \pm 2.09$ & $20 \pm 2.09$ & $12 \pm 1.89$ & $22 \pm 1.89$ & $30 \pm 2.09$ & $19 \pm 1.29$ & $22 \pm 2.89$ \\
\hline \multicolumn{13}{|c|}{ LSD at $p=0.05$} \\
\hline \multicolumn{13}{|c|}{ Between strains 4.427 .475 .14} \\
\hline \multicolumn{13}{|c|}{ Between treatments 3.835 .283 .63} \\
\hline
\end{tabular}

$\mathrm{mM} \mathrm{NaCl}$, while $H$. aquamarina (RT2) caused $(183 \%)$ increase at $50 \mathrm{mM} \mathrm{NaCl}$ in proline content. Inoculation of strain $H$. meridiana (PAa6) caused $224 \%$ increase at $200 \mathrm{mM} \mathrm{NaCl}$, while $H$. aquamarina (RT2) caused increase $187 \%$ at 200 $\mathrm{mM}$ increase in glycine betaine content of the plants. Exogenously applied osmolytes further stimulated the accumulation of osmolytes. It was observed that addition of glycine betaine reduced the endogenous level of proline when applied in combination with bacterial inoculation and also reduced the choline level.

\section{DISCUSSION}

The results in the present study supports previous reports showing that bacterial growth and osmolyte accumulation are strongly affected by varying media and salt concentrations (Roberts, 2005; Kouas et al., 2010). M9 medium and higher salt stress resulted in reduced growth of both strains. Association of increased osmolytes accumulation at higher salt stress suggests the possibility that osmolytes have a protective role in stabilizing macromolecules to extreme conditions and thus regulating metabolic activities of cells (Munns, 2002). Recently, accumulation of osmolytes and bacterial growth has been reported to be effected by culture conditions (Fallet et al., 2010). However, the influence of salt stress was not same for bacterial growth and osmolyte accumulation. Also, it is found that endogenous accumulation of glycine betaine is more than accumulated proline and choline at same culture conditions. The osmoprotective role of glycine betaine has been discussed previously (Zhao et al., 2009). There are many other evidences where 


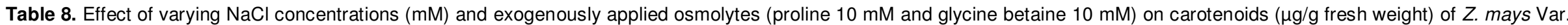
EV90.

\begin{tabular}{|c|c|c|c|c|c|c|c|c|c|c|c|c|}
\hline \multirow{3}{*}{ Treatment } & \multicolumn{12}{|c|}{ Carotenoids ( $\mu \mathrm{g} / \mathrm{g}$ fresh weight) } \\
\hline & \multicolumn{4}{|c|}{ Salt concentration (mM) } & \multicolumn{4}{|c|}{ Salt concentration $(\mathrm{mM})+10 \mathrm{mM}$ proline } & \multicolumn{4}{|c|}{ Salt concentration $(\mathrm{mM})+10 \mathrm{mM}$ glycine betaine } \\
\hline & 0 & 50 & 100 & 200 & 0 & 50 & 100 & 200 & 0 & 50 & 100 & 200 \\
\hline Control & $2.09 \pm 0.8$ & $2.1 \pm 1.23$ & $3.0 \pm 1.23$ & $2.0 \pm 1.23$ & $2.09 \pm 0.3$ & $22 \pm 1.09$ & $12 \pm 1.03$ & $12 \pm 1.7$ & $4.05 \pm 0.3$ & $23 \pm 3.09$ & $32 \pm 2.03$ & $42 \pm 1.88$ \\
\hline PAa6 & $5.09 \pm 0.3$ & $4.0 \pm 1.23$ & $4.0 \pm 1.23$ & $3.0 \pm 1.23$ & $12.1 \pm 0.3$ & $89 \pm 1.09$ & $65 \pm 1.03$ & $42 \pm 1.67$ & $8.12 \pm 0.9$ & $112 \pm 3.09$ & $89 \pm 1.93$ & $65 \pm 1.87$ \\
\hline RT-2 & $2.8 \pm 0.12$ & $3.1 \pm 2.23$ & $2.8 \pm 2.23$ & $1.2 \pm 2.23$ & $3.85 \pm 1.7$ & $65 \pm 3.23$ & $45 \pm 2.29$ & $32 \pm 2.93$ & $4.52 \pm 1.2$ & $75 \pm 3.93$ & $65 \pm 2.19$ & $65 \pm 2.03$ \\
\hline
\end{tabular}

LSD at $p=0.05$

Between strains 1.58 23.27 34.17

Between treatments 1.374 16.45 24.16

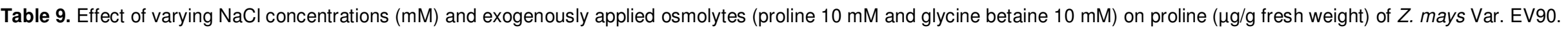

\begin{tabular}{|c|c|c|c|c|c|c|c|c|c|c|c|c|}
\hline \multirow{3}{*}{ Treatment } & \multicolumn{12}{|c|}{ Proline ( $\mu \mathrm{g} / \mathrm{g}$ fresh weight) } \\
\hline & \multicolumn{4}{|c|}{ Salt concentration (mM) } & \multicolumn{4}{|c|}{ Salt concentration $(\mathrm{mM})+10 \mathrm{mM}$ proline } & \multicolumn{4}{|c|}{ Salt concentration $(\mathrm{mM})+10 \mathrm{mM}$ glycine betaine } \\
\hline & 0 & 50 & 100 & 200 & 0 & 50 & 100 & 200 & 0 & 50 & 100 & 200 \\
\hline Control & $100 \pm 1.23$ & $109 \pm 9.89$ & $209 \pm 7.8$ & $234 \pm 9.8$ & $56 \pm 5.23$ & $78 \pm 12.89$ & $100 \pm 7.8$ & $123 \pm 12$ & $123 \pm 10.3$ & $234 \pm 9.89$ & $256 \pm 12.9$ & $312 \pm 23.8$ \\
\hline PAa6 & $209 \pm 0.98$ & $423 \pm 12.9$ & $489 \pm 12$ & $600 \pm 9.8$ & $120 \pm 11.9$ & $189 \pm 22.9$ & $200 \pm 5.2$ & $200 \pm 12.8$ & $289 \pm 12.8$ & $312 \pm 12.9$ & $332 \pm 19$ & $345 \pm 12.8$ \\
\hline RT-2 & $189 \pm 1.23$ & $309 \pm 12.2$ & $509 \pm 7.8$ & $765 \pm 3.8$ & $100 \pm 10.2$ & $200 \pm 32.2$ & $189 \pm 9.8$ & $198 \pm 5.8$ & $200 \pm 18.3$ & $298 \pm 12.2$ & $300 \pm 12$ & $345 \pm 23.8$ \\
\hline
\end{tabular}

LSD at $p=0.05$

Between strains 176.1262 .28126

Between treatments 152.5344 .0489 .35

endogenous proline and glycine betaine accumulation at higher salt concentrations have osmoprotective role coping with increasing salt concentrations (Munns, 2002; Nagata et al. 2009). It is also relevant to mention that bacterial growth require-ments are increased in the presence of salt stress so bacteria prefer LB medium for optimum growth and osmolytes accumulation unlike minimal media (Gouffi and Blanco, 2000).

Following this microbial work, we tested the comparative effect of bacterial inoculations and exogenously applied osmolytes on the salt tolerance of maize seedlings at 50,100 and 200 $\mathrm{mM} \mathrm{NaCl}$ concentrations separately along with their simultaneous application to seedlings. Increasing salt stress inhibited all growth parameters of plants. Moreover, plant growth at higher level of salinity showed significant reduction in germination, length, weight and chlorophyll contents. However, accumulation of osmolytes was higher in the plants grown at increasing salt concentrations. In general, it is accepted that salt sensitive plants are unable to compartmentalize the salt in vacuoles (Ashraf and Harris, 2004). It reduces the amount of available water needed for essential metabolic activities and ultimately causes yellowing of leaves, reducing photosynthetic activities and reduction in plant growth (Hussain et al., 2010). Decrease in fresh weight and increase in dry weight at high salinity is due to accumulation of inorganic ions $\left(\mathrm{Na}^{+} / \mathrm{K}^{+}\right.$ ions). The increased level of endogenous compatible solutes has also been reported to be involved with osmotic adjustment mechanism of plants (Ashraf and Harris, 2004).

Bacterial inoculation improved the plant growth and elevated their endogenous osmolytes accumulation at higher salt stress. Bacterial 


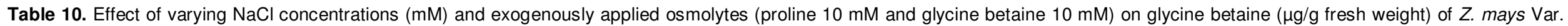
EV90.

\begin{tabular}{|c|c|c|c|c|c|c|c|c|c|c|c|c|}
\hline \multirow{3}{*}{ Treatment } & \multicolumn{12}{|c|}{ Glycine betaine ( $\mu \mathrm{g} / \mathrm{g}$ fresh weight) } \\
\hline & \multicolumn{4}{|c|}{ Salt concentrations $(\mathrm{mM})$} & \multicolumn{4}{|c|}{ Salt concentrations $(\mathrm{mM})+10 \mathrm{mM}$ proline } & \multicolumn{4}{|c|}{ Salt concentrations $(\mathrm{mM})+10 \mathrm{mM}$ glycine betaine } \\
\hline & 0 & 50 & 100 & 200 & 0 & 50 & 100 & 200 & 0 & 50 & 100 & 200 \\
\hline Control & $23 \pm 9.89$ & $45 \pm 12$ & $69 \pm 9.8$ & $89 \pm 9$ & $123 \pm 23.9$ & $134 \pm 12$ & $234 \pm 9.8$ & $298 \pm 9$ & $156 \pm 9.89$ & $165 \pm 12$ & $345 \pm 9.8$ & $389 \pm 9$ \\
\hline PAa6 & $100 \pm 8.78$ & $145 \pm 12$ & $156 \pm 8.8$ & $289.98 \pm 9$ & $300 \pm 12.78$ & $378 \pm 8.8$ & $435 \pm 12$ & $534 \pm 15$ & $245 \pm 8.78$ & $565 \pm 8.8$ & $600 \pm 12$ & $678 \pm 15$ \\
\hline RT-2 & $87 \pm 6.09$ & $90 \pm 12$ & $209 \pm 23$ & $256 \pm 23$ & $278 \pm 12.09$ & $367 \pm 12$ & $423 \pm 23$ & $512 \pm 23$ & $224 \pm 6.09$ & $389 \pm 12$ & $509 \pm 23$ & $645 \pm 23$ \\
\hline
\end{tabular}

LSD at $p=0.05$

Between strains 85.930 .0274

Between treatments 74.4721 .2252 .6

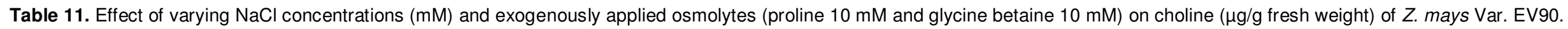

\begin{tabular}{|c|c|c|c|c|c|c|c|c|c|c|c|c|}
\hline \multirow{3}{*}{ Treatment } & \multicolumn{12}{|c|}{ Choline ( $\mu \mathrm{g} / \mathrm{g}$ fresh weight) } \\
\hline & \multicolumn{4}{|c|}{ Salt concentration (mM) } & \multicolumn{4}{|c|}{ Salt concentration $(\mathrm{mM})+10 \mathrm{mM}$ proline } & \multicolumn{4}{|c|}{$\begin{array}{c}\text { Salt concentration }(\mathrm{mM}) \\
\text { betaine }\end{array}$} \\
\hline & 0 & 50 & 100 & 200 & 0 & 50 & 100 & 200 & 0 & 50 & 100 & 200 \\
\hline PAa6 & $57 \pm 0.3$ & $123 \pm 12.23$ & $167 \pm 23$ & $209 \pm 10.93$ & $73 \pm 8.3$ & $89 \pm 9.23$ & $100 \pm 9.23$ & $109 \pm 12.23$ & $87 \pm 5.93$ & $96 \pm 10.23$ & $100 \pm 10.23$ & $112 \pm 8.23$ \\
\hline RT-2 & $39 \pm 0.12$ & $132 \pm 22.23$ & $123 \pm 3.23$ & $145 \pm 12.3$ & $56 \pm 9.12$ & $67 \pm 9.03$ & $100 \pm 8.23$ & $98 \pm 8.23$ & $65 \pm 8.12$ & $89 \pm 20.23$ & $100 \pm 8.23$ & $100 \pm 8.23$ \\
\hline
\end{tabular}

LSD at $p=0.05$

Between strains 3.1031 .18622

Between treatments 2.68 22.052 15.95

inoculations helped the plants to survive under salt stress due to better osmotic adjustment of plants (Nemati et al., 2011). The results obtained in the present study are also in agreement by many other previous and recent reports (Al-Sobhi, 2006; Jaleel et al, 2008; Chookhampaeng, 2011). Similar results were noted with fresh and dry weight where bacterial inoculations enhanced the biomass production that in turn increased the weight of plant (Rajkumar et al, 2009). Previous studies were also in agreement with the fact that increasing salt stress causes accumulation of proline and glycine betaine in germinating seeds (Gul and Khan, 2008; Nawaz and Ashraf, 2010).

We also evaluated the effect of exogenous osmolytes on the growth of plants. However, simultaneous application of exogenous osmolytes and bacterial inoculation significantly improved the plant growth parameters. Application of exogenously applied glycine betaine $(10 \mathrm{mM})$ was more effective than proline in alleviating salt stress for plant growth. There are also a number of other studies reporting proline and glycine betaine to increase the tolerance level of plants at high levels of salinity acting as an osmoregulator in the cytoplasm or as an osmoprotectant of proteins (Gul and khan, 2008). The exogenous application of choline chloride (Table 11) had nonsignificant effects as compared to proline and glycine betaine treated plants. It may also be speculated from our results that improved plant growth and photosynthetic activity is favoured by inoculation. Endogenous glycine betaine and 
proline, either taken from the soil, or up regulated by bacteria is one of the additional factors that may have contributed to a higher germination and other growth parameter under saline conditions. It is noteworthy that endogenous level of proline was higher in control seedlings treated with glycine betaine in the soil compared with those control seedlings treated with proline (Table 9).

Although, accumulation of glycine betaine as an osmoprotectant has been variously described, but under salt stress plants also accumulate proline as an osmoprotectant to overcome soil salinity. Plants treated with exogenous proline showed reduced level of accumulation of proline.

Previous studies are also in line with this finding, where proline overproduction in bacteria and plants has been found to reduce the level of proline accumulation due to feedback inhibition (Chen et al., 2007).

It is evident from the aforementioned results that plants accumulate osmolytes to cope with $\mathrm{NaCl}$ stress to a variable extent. This variable accumulation is not enough to maintain their osmotic potential under salt stress. From the results, it is also clear that endogenous level of osmolytes or both proline and glycine betaine were increased in plants treated with exogenously applied glycine betaine and proline. Accumulation of endogenous glycine betaine was more than accumulated proline. The increased accumulation of glycine betaine at higher salt stress indicates salt tolerance status of plants (Nawaz and Ashraf, 2010). It may be speculated that bacterial inoculation to plants might be involved in causing upregulation of osmolyte biosynthesis that tends to keep osmolytes level higher in plant cells. It was also observed that the higher the endogenous osmolytes accumulation, the lesser the inhibitory effects of salt on the growth parameters of plants, and as a result, seeds germinate as if they were at the lower levels of the $\mathrm{NaCl}$ (Ashraf and Harris, 2004)..

In view of the discussion, it is evident that osmolytes accumulation in bacteria and plants helps to alleviate salt stress. Accumulation of osmolytes to alleviate salinity in agricultural crops can be increased by exogenous osmolytes like proline and glycine betaine. However, increased demand of these osmolytes in future will not be economical for sustainable agriculture. Inoculated plants also have high capacity for osmolyte accumulation. Both strains $H$. meridiana (PAa6) and $H$. aquamarina (RT2) strains can accumulate osmolytes and exert stimulatory effects on the plant growth. Thus, these strains can be effective and suitable candidate for plant growth improvement, hence becoming an alternate economical approach for increasing crop productivity.

\section{ACKNOWLEDEGMENT}

The research work was financially supported by Higher Education Commission of Pakistan.

\section{REFERENCES}

Afrasayab S, Faisal M, Hasnain S (2010). Comparative study of wild and transformed salt tolerant bacterial strains on Triticum aestivum growth under salt stress. Braz. J. Microbiol. 41: 946-955.

Afrasayab S, Hasnain S (2000a). Synergistic growth stimulatory effects of mixed culture bacterial inoculations on the early growth of Triticum aestivum under $\mathrm{NaCl}$ stress. Pak. J. Biol. Sci. 16: 1016-1023.

Afrasayab S, Hasnain S (2000b). Early growth responses of Triticum aestivum Var Inqlab 91 under salt stress after inoculation with mixed culture rhizoplane and phylloplane salt tolerant bacteria. Sci. Int. 12: 79-86.

Ahmed A, Hasnain S (2010). Auxin-producing Bacillus sp.: Auxin quantification and effect on the growth of Solanum tuberosum. Pure Appl. Chem. 82(1): 313-319.

Al-Sobhi OA, Al-Zahrani HS, Al-Ahmadi ?? (Indicate Initials) (2006). Effect of Salinity on Chlorophyll \& Carbohydrate Contents of Calotropis procera Seedlings, Sci. J. King Faisal Univ. Basic Appl. Sci. 7: 105-114.

Ashraf M, Harris PJC (2004). Potential biochemical indicators of salinity tolerance in plants. Plant Sci. 166: 3-16.

Canovas D, Vargas C, Csonka LN, Ventosa A, Nieto JJ (1998). Synthesis of glycine betaine from exogenous choline in the moderately halophilic bacterium Halomonas elongata. Appl. Environ. Microbiol. 64: 4095-4097.

Chen M, Wei H, Cao J, Liu R, Wang Y, Zheng C (2007). Expression of Bacillus subtilis proBA genes and reduction of feedback inhibition of proline synthesis increases proline production and confers osmotolerance in transgenic Arabidopsis. J. Biochem. Mol. Biol. 40(3): 396-403.

Chookhampaeng $S$ (2011). The effect of salt stress on growth, chlorophyll content proline content and antioxidative enzymes of pepper (Capsicum annuum I.) seed. J Sci. Res. 49(1): 103-109.

Fallet C, Rohe P, Franco-Lara E (2010). Process optimization of the integrated synthesis and secretion of ectoine and hydroxyectoine under hyper/hypo-osmotic stress. Biotechnol. Bioeng. 107: 124-133.

Gerhardt GG, Murray RGE, Wood WA, Kreig NR (1994). Methods for general and molecular bacteriology, American Society for Microbiology, 1325. Massachusetts Ave., N. W. Washington, D.C. 2005.

Gouffi K, Blanco C (2000). Is the accumulation of osmoprotectant the unique mechanism involved in bacterial osmoprotection? Int. J. Food Microbiol. 55: 171-174.

Grieve CM, Grattan SR (1983). Rapid assay for determination of water soluble quaternary ammonium compounds. Plant and Soil, 70: 303307.

Gul B, khan MA (2008). Effect of compatible osmotica and plant growth regulators in alleviating salinity stress on the seed germination of Allenrolfea occidentalis. Pak. J. Bot. 40(5): 1957-1964.

Hirsch AM (2010). How rhizobia survive in the absence of a legume host, a stressful world indeed, in: Seckbach J, Grube M (Eds.), Symbiosis and Stress: Joint Ventures in Biology, Cellular Origin, Life in Extreme Habitats Astrobiol. 17(4): 375-391.

Hussain K, Nisar MF, Majeed A, Nawaz K, Bhatti KH, Afghan S, Shahazad A, Zia-ul-Hussnian S (2010). What molecular mechanism is adapted by plants during salt stress tolerance? Afr. J. Biotechnol. 9(4): 416-422.

Jadhav GG, Salunkhe DS, Nerkar DP, Bhadekar R K (2010). Isolation and characterization of salt-tolerant nitrogen-fixing microorganisms from food. J. Eur. Asia. Biol. Sci. 4: 33-40.

Jaleel CA, Sankar B, Sriaharan R, Panneerselvam R (2008). Soil salinity alters growth, chlorophyll content, and secondary metabolite accumulation in Catharanthus roseus, Turk. J. Biol. 32: 79-83.

Kahn M, Kolter R, Thomas C, Figurski D, Meyer R, Ramant E, Helinski DR (1979). Plasmid cloning vehicles derived from plasmid colE1, R6K \& RH4, In: Methods in Enzymology, (Wu R, Ed.,) Academic Press, New york. 86: 268-280.

Karlidag H, Esitken A, Yildirim EMF, Donmez Turan M (2011). Effects of plant growth promoting bacteria on yield, growth, leaf water content, membrane permeability, and ionic composition of strawberry under saline conditions. J. Plant Nutr. 34: 34-45.

Kouas S, Slatni T, Salah IB, Abdelly C (2010). Eco-physiological 
responses and symbiotic nitrogen fixation capacity of salt-exposed Hedysarum carnosum plants. Afr. J. Biotechnol. 9(44): 7462-7469.

Lichtenthaler HK, Wellburn AR (1983). Determination of total carotenoids and chlorophyll $\mathrm{a}$ and $\mathrm{b}$ pigments of leaf extracts in different solvents. Biochem. Soc. 11: 591-592.

Maas EV, Hoffman GJ, Chaba GD, Poss JA, Shannon MC (1983). Salt sensitivity of corn at various growth stages. Irrig. Sci. 4(1): 45-57. Mehr S, Afrasayab S, Hasnain S (2002). Effect of bacterial monoculture inoculations on the early growth of Triticum aestivum Var Inqlab 91 under $\mathrm{NaCl}$ stress. Pak. J. Biol. Sci. 5(6): 643-647.

Mirza TZ, Sabri AN, Hasnain S (1998). Salt tolerant bacteria from rhizosphere, rhizoplane histoplane and phylloplane of Mazus plant inhabitant of salt range. Sci. Int. 10: 151-156.

Munns R (2002). Comparative physiology of salt and water stress. Plant Cell Environ. 25: 239-250.

Nagata S, Wang Y, Zhang H, Sasaki H, Oshima A, Ishida I (2009). Effect of moderate salinity stress treatment on the stimulation of proline uptake and growth in Escherichia coli $\mathrm{C}_{\mathrm{s}} \mathrm{H}_{4}$ and its mutants under high salinity. J. Biosci. Bioeng. 108(3): 205-210.

Nasim M, Qureshi RH, Aziz T, Saqib M, Nawaz S, Sahi ST, Pervaiz S (2008). Growth and ionic composition of salt-stressed Eucalyptus camaldulensis and Eucalyptus tereticornis. Pak. J. Bot. 40(2): 799805.

Nawaz K, Ashraf MJ (2010). Exogenous Application of glycine betaine modulates activities of antioxidants in Maize Plants Subjected to Salt Stress. J. Agron. Crop Sci. 196(1): 28.

Nemati FM, Gholizadeh S, Esmaeili MA, Bihamta MR (2011). The effect of salinity stress on ions and soluble sugars distribution in leaves, leaf sheaths and roots of rice (Oryza sativa L.) seedlings. Plant Soil. Environ. 57(1): 26-33.
Rajkumar M, Prasad MNV, Freitas H, Ae N (2009). Biotechnological applications of serpentine soil bacteria for phytoremediation of trace metals. Crit. Rev. Biotechnol. 29: 120-30.

Roberts MF (2005). Organic compatible solutes of halotolerant and halophilic microorganisms. Saline Syst. 1: p. 5.

Sadowsky MJ (2005). Soil stress factors influencing symbiotic nitrogen fixation, in: Werner D and Newton WE (Eds.) Nitrogen Fixation Research in Agriculture, Forestry, Ecol. Environ. Springer, Dordrecht, The Netherlands. pp. 89-102.

Tonon G, Kevers C, Faivre-Rampant O, Graziani M, Gaspar T (2004). Effect of $\mathrm{NaCl}$ and mannitol iso-osmotic stresses on proline and free polyamine levels in embryogenic Fraxinus angustifolia callus. J. Plant Physiol. 161: 701-708.

Vyrides I, Stuckey DC (2009). Adaptation of anaerobic biomass to saline conditions: Role of compatible solutes and extracellular polysaccharides. Enzyme Microb. Tech. 44: 46-51.

Zhao X, Tan HJ, Liu YB, Li XR, Chen GX (2009). Effect of salt stress on growth and osmotic regulation in Thellungiella and Arabidopsis callus. Plant Cell. Tissue Org. 98(1): 97-103. 